\title{
Comparação entre Conduta Ativa com Ocitocina e Conduta Expectante na Rotura Prematura de Membranas em Gestações a Termo
}

\author{
Comparison between Active Management with Oxytocin and Expectant \\ Management for Premature Rupture of Membranes at Term
}

Ricardo Porto Tedesco, Nelson Lourenço Maia Filho

José Guilherme Cecatti, Renata Andreoni

\begin{abstract}
RESUM0
Objetivos: comparar, em uma população de gestantes brasileiras com rotura prematura de membranas a termo (RPM-T), as condutas expectante ou ativa com ocitocina.

Métodos: ensaio clínico prospectivo, randomizado e multicêntrico, avaliando as variáveis relativas ao tempo para o início do trabalho de parto e parto e tempo de internação hospitalar materna e neonatal. Foram selecionadas 200 gestantes com RPM-T, atendidas em quatro instituições públicas do estado de São Paulo de novembro de 1995 a fevereiro de 1997. As pacientes foram divididas aleatoriamente em dois grupos de conduta: ativa, com indução do trabalho de parto com ocitocina iniciando até $6 \mathrm{~h}$ de RPM, e expectante, aguardando-se o inicio espontâneo do trabalho de parto por um periodo máximo de $24 \mathrm{~h}$. Os dados foram analisados com o auxílio dos programas Epi-Info e SPSS-PC+, utilizando-se os testes estatísticos do $\chi^{2}$, t de Student e Log-rank.

Resultados: indicam que as diferenças entre a utilização da conduta ativa com ocitocina e a conduta expectante dizem respeito ao maior tempo necessário no grupo de conduta expectante para o início do trabalho de parto e parto, além da maior proporção de mulheres e recémnascidos com internação superior a três dias.

Conclusões: o tempo entre a admissão e o parto, o período de latência e o tempo entre a rotura das membranas e o parto foram maiores quando se adotou conduta expectante.
\end{abstract}

PALAVRAS-CHAVE: Rotura prematura de membranas. Indução de parto. Gestação de risco. Ocitocina.

Introdução

A rotura prematura de membranas é uma das entidades patológicas mais importantes em

\footnotetext{
Departamento de Tocoginecologia

Faculdade de Ciências Médicas

Universidade Estadual de Campinas

Correspondência:

José Guilherme Cecatti

DTG/CAISM/UNICAMP

Caixa Postal 6030

13081-970 - Campinas - SP - Fax: (019) 788-9304
}

Obstetrícia, com grande ocorrência em todo o mundo. Os índices de prevalência descritos são muito variáveis, entre os extremos de 1 a $20 \%$, refletindo provavelmente diferenças de definição, de população estudada, do tipo de estudo realizado e dos métodos diagnósticos empregados ${ }^{11}$.

Apesar da grande quantidade de fatores associados à sua ocorrência, a etiologia infecciosa é a mais provável. Recente revisão da literatura mostra que a prevalência de infecção subclínica da cavidade amniótica, em casos de RPM sem contração uterina, chega a quase $30 \%{ }^{17}$. O encontro de corioamnionite com membranas integras ${ }^{5}$ e de 
patógenos no líquido amniótico, idênticos aos da vagina $^{3}$ sugerem fortemente que a invasão bacteriana possa preceder a rotura das membranas e ser a sua causa.

Uma vez rotas as membranas, mesmo considerando a atividade bacteriostática e bactericida do líquido amniótico, a diminuição do seu volume poderia, então, associar-se a um maior risco de infecção. De fato, Vintzileos et al. ${ }^{21}$ observaram que, quanto menor o bolsão residual de líquido amniótico após a RPM, maior a incidência de infecção materna e fetal.

Os fatores que se relacionam à maior ou menor gravidade dessa e de outras complicações incluem a idade da gestação, o período de latência (tempo transcorrido entre a rotura das membranas e o início do trabalho de parto), tempo entre a rotura e a ultimação do parto, a manipulação do canal vaginal, o tipo de conduta assistencial utilizada, a associação com doenças maternas anteriores, a paridade e o tipo de apresentação fetal ${ }^{7}$.

A incidência de infecção intra-amniótica está diretamente relacionada ao período de latência que, por sua vez, apresenta relação inversa com a idade gestacional. Quanto menor for a idade gestacional, provavelmente maior será o período de latência ${ }^{25}$. Por essa razão, a corioamnionite é nitidamente mais comum em casos de rotura prematura pré-termo (idade gestacional menor que 37 semanas de gestação).

Com relação ao feto e ao recém-nascido, as complicações também variam amplamente, de acordo com a idade gestacional em que o evento ocorreu. Observa-se também maior gravidade no acometimento do produto das gestações pré-termo. Prematuridade, infecção perinatal e morbidade relacionadas ao oligoâmnio que geralmente se instala são as principais complicações que caracterizam uma gravidez, até então normal, como de alto risco, após a RPM. Quanto às complicações infecciosas, a RPM eleva significativamente o risco de infecção neonatal. Por todas essas razões, é possivel que a RPM seja, isoladamente, o diagnóstico que com maior freqüência requer a internação do recém-nascido em unidade de terapia intensiva ${ }^{6}$.

No grupo de mulheres com rotura prematura de membranas, a termo, não existindo os riscos da prematuridade, o período de latência assume especial importância na evolução natural dessa entidade patológica. A maioria das gestantes que não entrar em trabalho de parto em seis horas, irá fazê-lo em 12 horas; e a maioria daquelas que não entrarem em 12 horas, irá fazê-lo em até 18 horas. Apenas uma minoria das gestantes que não entrar em trabalho de parto em até 18 horas, irá fazê-lo dentro de 24 horas ou mais após a rotura ${ }^{25}$.
Diante desse comportamento, a abordagem terapêutica dos casos de RPM-T, antes do início espontâneo do trabalho de parto, tornou-se um dilema na prática obstétrica atual. Nos últimos 25 anos, o tratamento padrão utilizado nas principais entidades médicas dos Estados Unidos, para os casos de RPM-T, tem sido realizar a indução com ocitocina, caso o trabalho de parto não inicie espontaneamente entre 6 e 12 horas após a rotura de membranas ${ }^{25}$. Essa é a conduta médica que tem sido adotada na maioria dos serviços especializados no Brasil. É grande o número de autores que considera a conduta ativa com ocitocina a melhor opção terapêutica para os casos de RPM-T ${ }^{18,19,22}$.

Recentemente Hannah et al. ${ }^{8}$ mostraram resultados obtidos após a avaliação de mais de 5.000 gestantes com RPM-T, submetidas a diferentes tipos de abordagem terapêutica. No grupo submetido à indução imediata do trabalho de parto com ocitocina, observaram menor incidência de infecção materna e maior aceitação por parte das pacientes.

Nessa situação, entretanto, parece haver um aumento significativo da incidência de cesarianas ${ }^{16}$. É provável que a imaturidade do colo uterino seja o principal responsável pela falha de resposta à indução imediata do trabalho de parto com ocitocina $^{9,10}$. A avaliação das condições do colo uterino poderia ficar prejudicada nos casos de RPM, uma vez que se recomenda sua realização exclusivamente por meio do exame especular.

Procurando obter conduta segura e eficaz para os casos de RPM-T, alguns autores propõem a adoção de abordagem menos agressiva que a indução imediata do trabalho de parto, esperando pelo menos $24 \mathrm{~h}$ pelo início espontâneo do trabalho de parto. Muitos são os estudos que procuraram avaliar as possíveis vantagens da conduta expectante sobre a conduta ativa. Dentre eles, pelo fato de terem sido estudos prospectivos e controlados, destacam-se os trabalhos de Duff et al. ${ }^{4}$ e Morales e Lazar ${ }^{12}$. Ambos apontam para a diminuição significativa na indicação de cesarianas e na prevalência de corioamnionite, no grupo tratado de forma expectante.

Analisando-se a história natural da RPM-T, é fácil compreender porque a proposta de uma abordagem expectante poderia representar uma boa opção de conduta, talvez melhor que a indução com ocitocina: nesse período de $24 \mathrm{~h}$ a maioria das gestantes entraria em trabalho de parto, sem um aumento importante de infecção.

A despeito da alta incidência de RPM-T, não existem ainda muitos estudos aleatórios, envolvendo amostragem significativa de casos, para que os resultados encontrados possam ser irrestritamente generalizados. Isso torna a conduta 
expectante ainda carente de completo e definitivo embasamento científico, para que os níveis de segurança materna e perinatal necessários sejam estabelecidos.

Outro aspecto relevante refere-se ao custo com cada uma dessas condutas, ativa e expectante. Supõe-se que, embora possa haver um aumento do número de horas de internação para o grupo de gestantes submetido à conduta expectante, o seu custo deve ser significativamente inferior ao da conduta ativa com ocitocina.

É provável que uma outra alternativa segura para a indução do trabalho de parto nas gestantes com RPM-T, principalmente naquelas com o colo uterino desfavorável, seja o uso de metil análogo da prostaglandina E2 ou E1 para melhorar a maturação do colo uterino ou mesmo induzir o trabalho de parto. Muitos estudos têm demonstrado bons resultados com o uso dessas substâncias ${ }^{1,2,10,11,13,16,23}$. No Brasil, até o momento, não se dispõem dessas prostaglandinas para uso em obstetrícia, provavelmente por sua facilitação à prática do aborto induzido. A única prostaglandina que era disponivel no Brasil, apenas para uso hospitalar, o metil análogo da PGE 1 (misoprostol), apresenta vantagens em comparação com as prostaglandinas naturais ${ }^{24}$, entretanto, foi recentemente retirada do mercado.

Com o objetivo de verificar se, numa população de gestantes brasileiras de baixa renda, essa espera de 24 horas, após a RPM-T, permite efetivamente melhores resultados maternos e perinatais, em comparação com a indução imediata com ocitocina, realizou-se este estudo prospectivo.

Espera-se que os resultados obtidos possam contribuir para o melhor conhecimento dessas duas possibilidades terapêuticas para os casos de RPM-T, objetivando-se a redução da necessidade de realização de tratamentos invasivos e dispendiosos, para que se possa melhorar os indicadores de saúde materno-infantil da população brasileira, em uma patologia obstétrica tão importante e freqüente como é a RPM.

\section{Pacientes e Métodos}

O estudo cujos dados parciais são apresentados neste artigo foi um ensaio clínico controlado, aleatório e multicêntrico, realizado nas seguintes instituições hospitalares públicas do estado de São Paulo: Hospital de Clínicas Especializadas de Franco da Rocha; Hospital de Caridade São Vicente de Paula, Jundiaí; Hospital e Maternidade Leonor Mendes de Barros, São Paulo e Centro de Assistência Integral à Saúde da Mulher, Campinas. O estudo foi previamente aprovado pelo Comitê de Ética das instituições envolvidas.

O tamanho amostral calculado para o estudo foi de 98 mulheres por grupo ${ }^{14}$. Selecionaram-se então 200 gestantes com o diagnóstico de rotura prematura de membranas que cumpriram com os seguintes critérios de inclusão: confirmação da RPM até seis horas da ocorrência, gestação única a termo, apresentação cefálica, líquido amniótico claro e ausência de febre ou qualquer outro sinal indicativo de infecção ou sofrimento fetal. Foram excluídos os casos em trabalho de parto quando da admissão, com duas ou mais cesarianas ou contra-indicação para o parto vaginal, com diabetes, cardiopatia, hemorragia genital e anemia grave. As gestantes foram admitidas de novembro de 1995 a fevereiro de 1997.

Os casos foram distribuídas aleatoriamente, após o esclarecimento à mulher sobre o trabalho e sua concordância expressa em participar do estudo, por meio de uma tabela de números aleatórios, em uma ou outra das duas condutas sob investigação. A primeira, conduta expectante, correspondia à não-indução do trabalho de parto por um período de 24 horas após a rotura, logo a seguir iniciavase uma indução com infusão endovenosa de ocitocina em doses habituais. A segunda opção, a conduta ativa, correspondia à indução imediata do trabalho de parto com ocitocina, iniciada sempre antes de se completarem seis horas da rotura de membranas. Todas as mulheres foram avaliadas clínica e laboratorialmente, tiveram seu trabalho de parto acompanhado e a vitalidade fetal esporadicamente avaliada por meio de monitorização fetal eletrônica. $O$ toque vaginal foi realizado pela primeira vez apenas após iniciado o trabalho de parto.

No presente artigo são apresentados os resultados relativos ao intervalo entre a admissão e o parto, período de latência, intervalo da RPM ao parto, além do tempo de internação da mãe e do recém-nascido. Os resultados relativos às complicações maternas e perinatais, via de parto e vitalidade do recém-nascido são objetos de outra publicação ${ }^{20}$.

Os formulários referentes a cada caso foram cuidadosamente revisados por um dos investigadores, corrigidos quando necessário, e digitados para formar um banco de dados em EpiInfo. Para a análise, utilizou-se o teste t de Student para as diferenças de médias entre as variáveis numéricas (tempo), o teste do $\chi^{2}$ para as variáveis categorizadas e o teste de log-rank (Kaplan-Meyer) para a análise de sobrevivência relativa às taxas acumuladas de RPM até o parto. Utilizaram-se os pacotes Epi-Info e SPSS-PC para a análise estatística. 


\section{Resultados}

Foram incluídas no estudo 200 gestantes com RPM-T que preenchiam os critérios de inclusão e exclusão. Dessas, 106 foram aleatoriamente indicadas para conduta ativa e 94 para conduta expectante.

Com o objetivo de se conferir o processo de indicação aleatória dos grupos de tratamento, são apresentados os resultados relativos às variáveis de controle. A Tabela 1 mostra que, para os dois grupos, não houve diferença significativa na distribuição das gestantes com RPM-T, segundo grupos etários.

Tabela 1 - Distribuição das gestantes com RPM-T por grupos etários, segundo a conduta adotada.

\begin{tabular}{lcc} 
& \multicolumn{2}{c}{ Conduta } \\
Grupo etário (anos) & Ativa & Expectante \\
\hline Até 19 & 27 & 24 \\
20 a 24 & 29 & 34 \\
25 a 29 & 26 & 18 \\
30 a 34 & 14 & 11 \\
35 ou mais & 8 & 9 \\
Total (n) & 104 & 96 \\
\hline
\end{tabular}

$\chi^{2}=2,13 \quad p=0,71$

A Tabela 2 mostra que também não houve diferença significativa entre os grupos, segundo a paridade, número de gestações anteriores, antecedente de cesariana anterior, raça, escolaridade, idade gestacional, índice de Bishop ao primeiro toque vaginal após o início do trabalho de parto e tempo entre a RPM e a admissão.

Não se observaram também diferenças significativas entre os dois grupos de conduta, pela distribuição das gestantes por grupos de peso do recém-nascido (Tabela 3 ).

Cerca de dois terços dos partos ocorreram por via vaginal, de forma semelhante nos dois grupos. O número de cesarianas, entretanto, por falha de indução, foi significativamente maior no grupo da conduta ativa. Esses resultados, bem como os referentes à vitalidade neonatal, infecção materna e neonatal, todos sem diferença significativa entre os dois grupos, são objeto de outra publicação ${ }^{20}$.
Tabela 2 - Distribuição das gestantes com RPM-T, segundo algumas características de controle e conduta.

\begin{tabular}{|c|c|c|c|c|}
\hline \multirow[b]{2}{*}{ Características } & \multicolumn{2}{|c|}{ Conduta } & \multirow[b]{2}{*}{$\chi^{2}$} & \multirow[b]{2}{*}{$\mathbf{p}$} \\
\hline & Ativa & Expectante & & \\
\hline Nuliparidade & 55 & 47 & 0,31 & 0,579 \\
\hline 2 ou mais gestações anteriores & 59 & 50 & 0,43 & 0,509 \\
\hline 1 cesariana anterior & 11 & 17 & 2,11 & 0,146 \\
\hline Raça branca & 64 & 61 & 0,09 & 0,770 \\
\hline $\begin{array}{l}\text { Escolaridade até } 1^{\circ} \text { grau } \\
\text { completo }\end{array}$ & 90 & 84 & 0,04 & 0,839 \\
\hline IG até 39 semanas & 71 & 72 & 1,11 & 0,292 \\
\hline IB no $1^{\circ}$ toque $\leq 5$ & 48 & 47 & 0,16 & 0,691 \\
\hline RPM/admissão até $3 \mathrm{~h}$ & 65 & 51 & 1,80 & 0,179 \\
\hline Total (n) & 104 & 96 & & \\
\hline
\end{tabular}

IG: idade gestacional

IB: índice de Bishop

Tabela 3 - Distribuição das gestantes com RPM-T por peso do recém-nascido, segundo a conduta.

\begin{tabular}{|c|c|c|}
\hline \multirow[b]{2}{*}{ Peso em gramas } & \multicolumn{2}{|c|}{ Conduta } \\
\hline & Ativa & Expectante \\
\hline Até 2.499 & 6 & 3 \\
\hline $2.500-2.999$ & 38 & 37 \\
\hline $3.000-3.499$ & 41 & 37 \\
\hline$\geq 3.500$ & 19 & 19 \\
\hline Total (n) & 104 & 96 \\
\hline
\end{tabular}

A Tabela 4 resume as médias e os desvios padrão dos valores referentes às variáveis dependentes quantitativas, mostrando que houve diferença estatisticamente significativa entre os grupos de conduta, quando se analisou o tempo entre a admissão e o parto, o período de latência e o tempo entre a rotura das membranas e o parto.

Quando se considerou a distribuição das gestantes e recém-nascidos pelo tempo de internação hospitalar até 3 dias, esses números foram significativamente maiores para a conduta ativa (Tabela 5).

No grupo da conduta expectante, que teve um tempo de latência significativamente maior que o do grupo da conduta ativa, $85,4 \%$ das gestantes entraram em trabalho de parto espontâneo dentro 
das primeiras 24 horas. As demais $(14,6 \%)$ utilizaram ocitocina para a indução do mesmo. Dessas, 42,8\% (6 casos) não responderam a essa indução de maneira satisfatória, sendo submetidas a cesariana por falha de indução.

Tabela 4 - Média e desvio padrão dos valores referentes a algumas variáveis dependentes das gestantes com RPM-T, segundo a conduta.

\begin{tabular}{|c|c|c|c|c|c|}
\hline \multirow[b]{2}{*}{ Variáveis * } & \multicolumn{2}{|l|}{ Ativa } & \multicolumn{2}{|c|}{ Ativa } & \multirow[b]{2}{*}{$\mathbf{p}$} \\
\hline & média \pm & DP & média \pm & DP & \\
\hline Intervalo admissão-parto** & $11,89 \pm$ & 4,93 & $16,80 \pm$ & 9,25 & $<0,0001$ \\
\hline Período de latência** & $6,86 \pm$ & 2,96 & $11,25 \pm$ & 8,66 & $<0,0001$ \\
\hline Intervalo RPM-parto** & $15,03 \pm$ & 5,33 & $20,07 \pm$ & 9,56 & $<0,0001$ \\
\hline
\end{tabular}

Tabela 5 - Distribuição das gestantes com RPM-T, por tempo de internação hospitalar materna e do recém-nascido, segundo a conduta.

\begin{tabular}{|c|c|c|}
\hline \multirow[b]{2}{*}{ Internação (dias) } & \multicolumn{2}{|c|}{ Conduta } \\
\hline & Ativa & Expectante \\
\hline \multicolumn{3}{|c|}{ Do recém-nascido * } \\
\hline Até 3 & 89 & 69 \\
\hline$>4$ & 15 & 27 \\
\hline \multicolumn{3}{|l|}{ Materna** } \\
\hline Até 3 & 92 & 69 \\
\hline$>4$ & 12 & 27 \\
\hline Total (n) & 104 & 96 \\
\hline $\begin{aligned} * & \chi^{2}=5,65 \\
* \star & \chi^{2}=8,75\end{aligned}$ & & \\
\hline
\end{tabular}

Para cada tempo, as taxas de parto no grupo da conduta ativa foram significativamente maiores do que no grupo da conduta expectante. Isso está demonstrado na Figura 1 que mostra, graficamente, embora de uma maneira inversa, como os partos aconteceram mais precocemente no grupo da conduta ativa. Nesse grupo, a totalidade dos partos tinha acontecido após 32 horas de RPM, ao passo que no grupo expectante isso ocorreu com 48 horas de RPM.

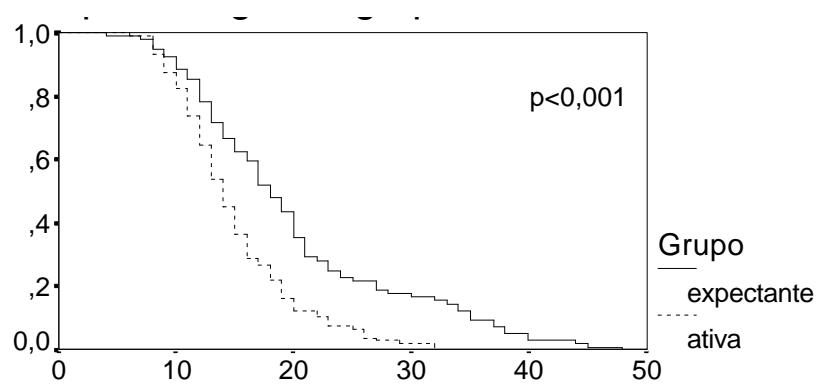

Figura 1 - Taxas acumuladas de RPM até o parto, segundo grupo de conduta.

\section{Discussão}

Os resultados desse estudo indicam haver diferenças significativas entre os grupos quanto ao período de latência, tempo entre a admissão hospitalar e o parto, e tempo entre a RPM e o parto, com valores maiores no grupo da conduta expectante. Também foram maiores na conduta expectante os períodos de internação hospitalar, tanto para as mulheres quanto para os recémnascidos.

No Brasil, até onde foi possível revisar a literatura, essa parece ser a primeira vez que se realiza um ensaio clínico aleatório, comparando duas possibilidades terapêuticas para o tratamento de gestantes com RPM-T. A conduta mais freqüentemente utilizada nas instituições brasileiras parece ser a ativa, com indução do trabalho de parto com ocitocina. Provavelmente, até então, nunca se realizou no país uma avaliação criteriosa da utilização da conduta expectante para o tratamento de gestantes com RPM-T.

Em linhas gerais, acredita-se que é permitida a adoção da conduta ativa ou espectante, desde que seja baixa a incidência de infecção nas situações de RPM-T quando tomadas as devidas precauções, com a adequada monitorização clínica e laboratorial da gestante, em nivel hospitalar e, principalmente, com a completa proscrição do toque vaginal antes do início do trabalho de parto. De fato, o índice de infecção materna e neonatal foi similar em ambos os grupos.

Uma avaliação de extrema importância poderia ser feita pela seleção de gestantes por grau de maturidade do colo uterino. É provável que existam gestantes que se beneficiem mais com uma 
ou outra opção terapêutica. A dificuldade está, no entanto, na forma de avaliação das condições do colo uterino, já que existem evidências suficientes para não recomendar o toque vaginal na RPM antes de iniciado o trabalho de parto.

As taxas de cesárea foram relativamente elevadas nos dois grupos, de 33\% e de 25\% para as condutas ativa e expectante, respectivamente, muito acima dos valores relatados em outros países $^{8}$, porém, muito inferiores à média populacional para o estado de São Paulo ${ }^{15}$.

Uma possivel limitação metodológica poderia ser a impossibilidade de se estabelecer uma postura médica uniforme, em estudos multicêntricos que envolvem grande número de médicos, com diferentes treinamentos. Embora tenha sido criado um protocolo padronizando em relação às duas modalidades terapêuticas estudadas, muitos aspectos relacionados à atitude médica são difíceis de serem padronizados, pois o julgamento clínico envolve certo grau de subjetividade. Um exemplo disso é a divergência existente em relação ao que se considera o momento mais adequado para se indicar uma cesariana

Em termos práticos, os resultados desse estudo indicam que não existem diferenças significativas na utilização de qualquer uma das duas possibilidades terapêuticas estudadas, além do maior tempo necessário para o início do trabalho de parto e parto na conduta expectante, também com o conseqüente aumento no tempo de internação materno e neonatal. A indução imediata do trabalho de parto com ocitocina apresenta a desvantagem, em relação à conduta expectante, de requerer a presença constante de profissional experiente para o tratamento de eventuais complicações e iatrogenias que dela possam advir.

Existe ainda a possibilidade de redução no custo do atendimento médico com a conduta expectante. Essa conduta, em geral, dispensa o uso de medicamentos durante o trabalho de parto, o que acarretaria em diminuição desse custo. Por outro lado, uma vez sendo mais prolongados os períodos de latência e para o parto na conduta expectante, também foram maiores o tempo de internação para as puérperas e recém-nascidos, com provável conseqüente elevação do custo hospitalar.

Em países desenvolvidos, o uso cada vez mais rotineiro e eficaz de prostaglandinas para essa indução tem mostrado bons resultados, principalmente nos casos nos quais o colo uterino apresenta-se imaturo ${ }^{10,13}$. Na avaliação de seu uso no tratamento de gestantes com RPM-T, alguns estudos compararam as prostaglandinas com a ocitocina, bem como o uso de ambas com a conduta expectante. Em geral, o que se observou foi que no grupo tratado com as prostaglandinas foram obtidos os melhores resultados ${ }^{16}$.

Com o objetivo de tornar mais acessivel e menos dispendiosa a indução do trabalho de parto com prostaglandinas, vários autores em diversos países têm utilizado, em lugar da prostaglandina $\mathrm{E} 2$, o misoprostol por via vaginal. Os resultados com sua utilização têm sido promissores, mostrando que essa substância poderá vir a ser uma alternativa segura em lugar das prostaglandinas naturais ${ }^{1,24}$.

No Brasil, o Ministério da Saúde ainda não aprovou a utilização obstétrica de nenhum tipo de prostaglandina. Seria muito oportuno se pudéssemos avaliar seu uso para a indução do trabalho de parto, sobretudo em situações específicas como a RPM-T. Uma boa opção seria o emprego do misoprostol, já que essa substância já foi comercializada no país, com outra finalidade, além de ter um custo muito menor que as prostaglandinas E2 e eficácia bastante elevada.

\section{Agradecimentos}

Os autores agradecem a colaboração de toda a equipe médica responsável pela condução dos casos do estudo nos hospitais participantes: Hospital de Clínicas Especializadas de Franco da Rocha, Hospital São Vicente de Paula em Jundiaí, Hospital e Maternidade Leonor Mendes de Barros, São Paulo e Centro de Assistência Integral à Saúde da Mulher, Campinas. Agradecem ainda ao Fundo de Apoio ao Ensino e Pesquisa (FAEP) da UNICAMP pelo suporte financeiro na realização do estudo.

\section{SUMMARY}

Objective: to compare the expectant versus active management with oxytocin in a Brazilian population of pregnant women with premature rupture of membranes (PROM) at term.

Methods: a prospective, randomized and multicenter clinical trial was performed, evaluating variables concerning the time from PROM until the onset of labor and delivery, and maternal and neonatal hospitalization periods. Two hundred pregnant women with PROM at term were selected from four public hospitals in São Paulo state, from November 1995 to February 1997. They were randomly divided into two groups: active management, with oxytocin induction of labor until $6 \mathrm{~h}$ of PROM; and expectant management, waiting for the spontaneous onset of labor up to $24 \mathrm{~h}$. The data were analyzed with the Epi-Info and SPSS-PC+ packages, using the statistical $\chi^{2}$, Student's $t$ and log-rank tests.

Results: the results indicate that the differences between the two managements concern to the longer time needed for the 
expectant management group until onset of labor and delivery, besides the higher number of women and neonates who remained in hospital for more than three days.

Conclusions: the time between admission and onset of labor and delivery, and also the latent period were longer in the expectant management group.

KEY WORDS: Rupture of membranes, premature. Labor induction. Risk pregnancy. Oxytocin.

\section{Referências}

1. Bugalho A, Bique C, Machungo F, Faúndes A. Lowdose vaginal misoprostol for induction of labor with a live fetus. Int J Gynaecol Obstet 1995; 49: 149-55.

2. Chua S, Arulkumaran S, Kurup A, Anandakumar C, Tay D, Ratnan SS. Does prostaglandin confer significant advantage over oxytocin infusion for nulliparas with pre-labor rupture of membranes at term? Obstet Gynecol 1991; 77: 664-7.

3. Creatsas G, Pavlatos M, Lolis D, Aravantinos D, Kaskarelis D. Bacterial contamination of the cervix and premature rupture of membranes. Am J Obstet Gynecol 1981; 139: 522-5.

4. Duff P, Huff RW, Gibbs RS. Management of premature rupture of membranes and unfavorable cervix in term pregnancy. Obstet Gynecol 1984; 63: 697-702.

5. Faro S, Walker C, Pierson RL. Amnionitis with intact amniotic membranes involving Streptobacillus moniliformis. Obstet Gynecol 1980; 55(3 suppl): 9S-11S.

6. Garite TJ, Keegan KA, Freeman RK, Nageotte MP. A randomized trial of ritodrine tocolysis versus expectant management in patients with premature rupture of membranes at 25 to 30 weeks gestation. Am J Obstet Gynecol 1987; 157: 388-93.

7. Gunn GC, Mishell DR Jr, Morton DG. Premature rupture of the fetal membranes. A review. Am J Obstet Gynecol 1970; 106: 469-83.

8. Hannah ME, Ohlsson A, Farine D, Hewson SA, Hodnett ED, Myhr TL, et al. Induction of labor compared with expectant management for prelabor rupture of the membranes at term. Termpron Study Group. N Eng J Med 1996; 334: 1005-10.

9. Hjertberg R, Hammarstrom M, Moberger B, Nordlander E, Granstrom L. Premature rupture of the membranes (PROM) at term in nulliparous women with a ripe cervix. A randomized trial of 12 or 24 hours of expectant management. Acta Obstet Gynecol Scand 1996; 75: 48-53.
10. Mahmood TA, Dick MJ, Smith NC, Templeton AA. Role of prostaglandin in the management of prelabour rupture of the membranes at term. Br J Obstet Gynaecol 1992; 99: 112-7.

11. Meikle SF, Bissell ME, Freedman WL, Gibbs RS. A retrospective review of the efficacy and safety of prostaglandin E2 with premature rupture of the membranes at term. Obstet Gynecol 1992; 80: 76-9.

12. Morales WJ, Lazar AJ. Expectant management of rupture of membranes at term. South Med $\mathbf{J}$ 1986; 79: 955-8.

13.Nimrod C, Varela-Gittings F, Machin G, Campbell $\mathrm{D}$, Wesenberg $\mathrm{R}$. The effect of very prolonged membrane rupture on fetal development. Am J Obstet Gynecol 1984; 148: 540-3.

14. Pocock SJ. Clinical trials: a practical approach. Chichester: John Wiley \& Sons; 1983.

15. Rattner D. Sobre a hipótese de estabilização das taxas de cesárea do Estado de São Paulo, Brasil. Rev Saúde Pública 1996; 30: 19-33.

16. Ray DA, Garite TJ. Prostaglandin $\mathrm{E}_{2}$ for induction of labor in patients with premature rupture of membranes at term. Am J Obstet Gynecol 1992; 166: 836-43.

17. Romero R, Ghidini A, Mazor M, Behnke A. Microbial invasion of the amniotic cavity in premature rupture of membranes. Clin Obstet Gynecol 1991; 34: 769-78.

18. Sperling LS, Schantz AL, Wahlin A, Duun S, Jaszczak $\mathrm{P}$, Scherling B, et al. Management of prelabor rupture of membranes at term. A randomized study. Acta Obstet Gynecol Scand 1993; 72: 627-32.

19. Tamsen L, Lyrenãs S, Cnattingius S. Premature rupture of the membranes - intervention or not. Gynecol Obstet Invest 1990; 29: 128-31.

20. Tedesco RP. Conduta expectante versus ativa com ocitocina na rotura prematura de membranas a termo. Tese de Mestrado. Universidade Estadual de Campinas, 1977.

21. Vintzileos AM, Campbell WA, Nochimson DJ, Weinbaum PJ. Degree of oligohydramnios and pregnancy outcome in patients with premature rupture of the membranes. Obstet Gynecol 1985; 66: 162-7.

22. Wagner MV, Chin VP, Peters CJ, Drexler B, Newman LA. A comparison of early and delayed induction of labor with spontaneous rupture of membranes at term. Obstet Gynecol 1989; 74: 93-7.

23. Wing DA, Jones MM, Rahall A, Goodwin TM, Paul RH. A comparison of misoprostol and prostaglandin E2 gel for preinduction cervical ripening and labor induction. Am J Obstet Gynecol 1995; 172: 1804-10.

24. Wing DA, Rahall A, Jones MM, Goodwin M, Paul RH. Misoprostol: an effective agent for cervical ripening and labor induction. Am $\mathbf{J}$ Obstet Gynecol 1995; 172: 1811-6.

25. Zlatnik FJ. Conduta nos casos de ruptura prematura das membranas no termo. Clin Obstet Ginecol Am Norte 1992; 2: 383-94. 